\title{
Supramolecular Structure of Polypeptides in Concentrated Solutions and Films. II. Small-Angle Light Scattering from Cholesteric Mesophase
}

\author{
Takeji Hashimoto, Satoshi EbISU, * Nobuyuki InABA, ** \\ and Hiromichi KaWAI \\ Department of Polymer Chemistry, Faculty of Engineering, Kyoto University, \\ Yoshida, Sakyo-ku, Kyoto 606, Japan.
}

(Received December 9, 1980)

\begin{abstract}
The cholesteric mesophase of poly( $\gamma$-benzyl L-gultamate) in concentrated solutions of helicogenic solvents was investigated by the laser-light scattering technique. The observed, elastic scattering intensity distributions are theoretically explained in terms of the cholesteric domains embedded in an optically active medium. Analysis of the scattering patterns provides information on the identity period and sense of the cholesteric twisting.

KEY WORDS Cholesteric Liquid Crystal / Poly( $\gamma$-benzyl L-gultamate) /

Laser Light Scattering / Form Optical Rotation / Sense of Cholesteric

Twisting / Cholesteric Pitch /
\end{abstract}

The laser-light scattering technique has been developed to characterize the supramolecular structure of polymeric systems in solution and in the liquid and solid states. In this series of papers, we study the application of this technique to the supramolecular structure of polypeptides in concentrated solutions and in solids.

A number of reports have been published on the supramolecular structure of polymer liquid crystals. There are even a few papers dealing with the application of the scattering technique for analyzing the cholesteric pitch ${ }^{1,2}$ or to qualitatively the orientation correlations (which exist in solutions and in solids) and variation in these with solvent, film preparing conditions $\mathbf{s}^{3,4}$ and applied fields. ${ }^{5}$ Our main purpose in this paper is to gain some insight into the elastic light scattering behavior of cholesteric liquid crystals so as to further expand applicability of this technique.

We shall study, both theoretically and experimen-

* Present address: Central Research Laboratory, Sumitomo Chemical Co., Ltd:, 40, Tsukahara, Takatsuki 569, Japan.

** Present address: Resarch Center for Products Development, Idemitsu Petrochemical Co., Ltd., Kamiizumi, Sodegaura, Kimitsu, Chiba 292-01, Japan. tally, elastic light scattering from the cholesteric mesophase of polypeptides in concentrated solutions of helicogenic solvents. In EXPERIMENTAL section we discuss typical laser light scattering patterns from the cholesteric liquid crystals of poly $(\gamma$-benzyl L-glutamate) (PBLG). The scattering at very small momentum transfer $|\boldsymbol{q}|=$ $(4 \pi / \lambda) \sin \left(\theta^{\prime} / 2\right)(\lambda$, wavelength of light in medium; $\theta^{\prime}$, the scattering angle in the medium) depends on the shape of the cholesteric domain as a whole as will be described in THEORETICAL ANALYSIS section. At an intermediate momentum transfer, $|\boldsymbol{q}|=0(1 / S)$ where $S$ is one half of the cholesteric pitch $P$, the scattering intensity reaches a maximum. This scattering maximum is attributed to diffraction arising from the cholesteric pitch, ${ }^{1,2,6}$ the scattering vector $\boldsymbol{q}_{\mathrm{m}}$ of the maximum intensity and $S$ being interrelated by Bragg's equation,

$$
q_{\mathrm{m}}=2 \pi / S \text { or } 2 S \sin \left(\theta_{\mathrm{m}}{ }^{\prime} / 2\right)=\lambda
$$

In THEORETICAL ANALYSIS section, we give a theoretical interpretation of the observed "cholesteric scattering patterns" (i.e., the scattering arising from cholesteric twisted structure at $q \simeq q_{\mathrm{m}}$ ). We verify eq 1 and also find that the observed distortion of the "cholesteric scattering patterns" under $H_{\mathrm{v}}$ 
condition in terms of their dependencies on the azimuthal angle $\mu$ is due to the effect of "form optical rotation" 7 on the scattering patterns. ${ }^{6,8,9}$ The manner of distortion in the $H_{\mathrm{V}}$ patterns determines the sense of cholesteric twisting in the cholesteric liquid crystals (see THEORETICAL ANALYSIS section, 3) and the simultaneous determination of the sense and the pitch of the twisting is of value in making a systematic interpretation of the twisting mechanism as a function of concentration, temperature, and solvents (see DISCUSSION section).

\section{EXPERIMENTAL SECTION}

\section{Test Specimens and Apparatus}

PBLG samples were prepared by polymerizing $N$ carboxyanhydride of $\gamma$-benzyl L-gultamate in a mixture of dioxane-dichloromethane $(1: 1 \mathrm{v} / \mathrm{v})$ with triethylamine as an initiator. The polymers were further fractionated, and the samples used in this work have the heterogeneity index $\left(M_{w} / M_{n}\right)$ approximately equal to 1.3 from gel-permeation chromatography (GPC) measured on a dimethylformamide solution. The PBLG specimens have molecular weight of $1.0 \times 10^{5}$ according to viscosity measurements in dichloroacetic acid at $25^{\circ} \mathrm{C}$.

The PBLG specimens were dissolved into various helicogenic solvents such as $m$-cresol, 1,1,2,2-tetrachloroethane (TCE), dioxane, and dichloroethane (DCE). The solutions were enclosed in glass cells of $1 \mathrm{~mm}$ in thickness. The cell was placed in a temperature enclosure whose temperature was regulated within $\pm 0.25^{\circ} \mathrm{C}$.

Figure 1 shows a schematic diagram of the laser light-scattering apparatus used in this work. A plane-polarized laser beam (He-Ne laser of $20 \mathrm{~mW}$, $\lambda_{0}=6328 \AA$ ) was used as the incident source. The polarization direction of the beam was rotated by a polarization rotator (its essential part being a $\lambda_{0} / 2$ retardation plate). The sample cell of $10 \mathrm{~mm} \mathrm{di-}$ ameter and $1 \mathrm{~mm}$ thick was placed in a temperature enclosure mounted on a horizontal $x y$ stage. The scattered light intensity was recorded through an analyzer onto polaroid land pack films or by a TV camera system composed of a CCTV camera (silicone vidicon, NEC model TI-302A), Video Tape Recorder and TV Monitor and capable of recording 60 frames per second.

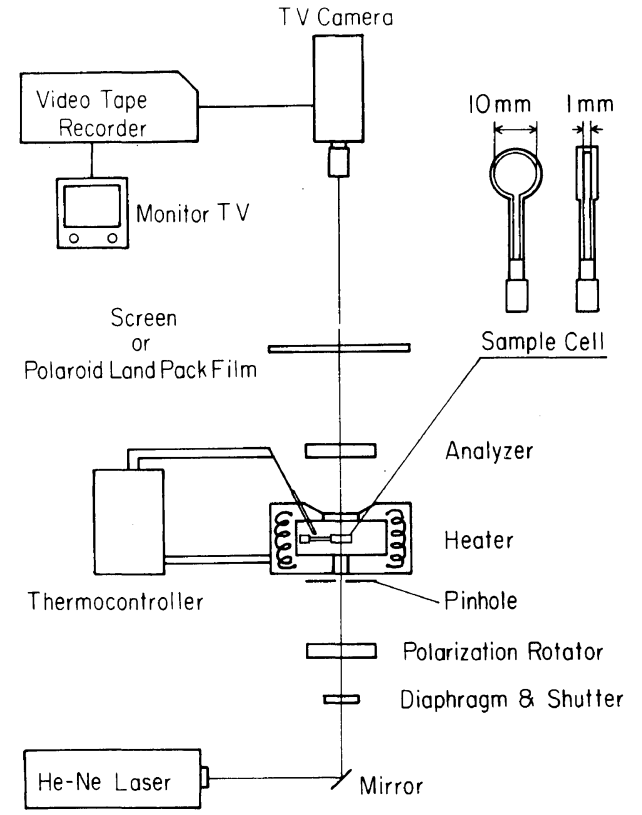

Figure 1. Schematic diagram of the laser-light scattering apparatus.

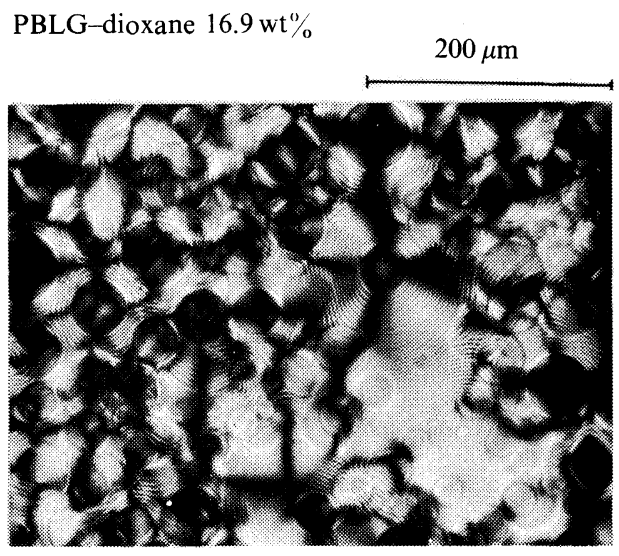

Figure 2. Typical cholesteric liquid-crystalline texture of PBLG observed under polarized light microscope (polarization directions are vertical and horizontal, $16.9 \mathrm{wt} \%$ dioxane solution).

\section{Results}

Figure 2 shows a typical cholesteric liquidcrystalline texture of PBLG observed by a polarized light microscopy ( $16.9 \mathrm{wt} \%$ dioxane solution). There is some confusion as to the concentration specifications in the literature on polypeptide liquid 
PBLG-dioxane $16.9 \mathrm{wt} \%$

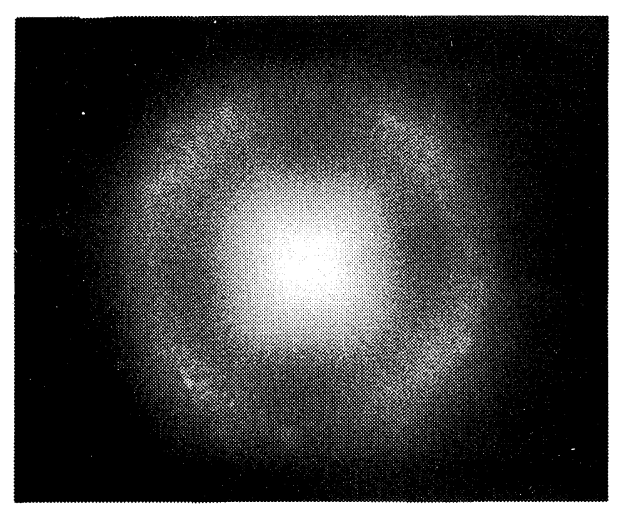

$H_{\mathrm{v}}$

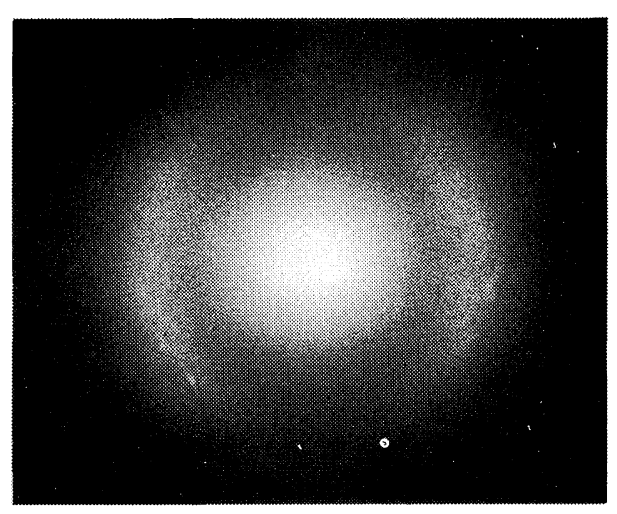

$V_{\mathrm{V}}$

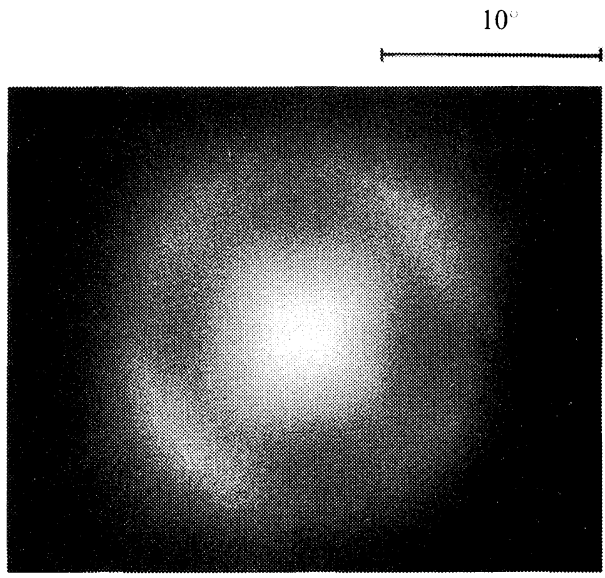

$V_{\mathrm{H}}$

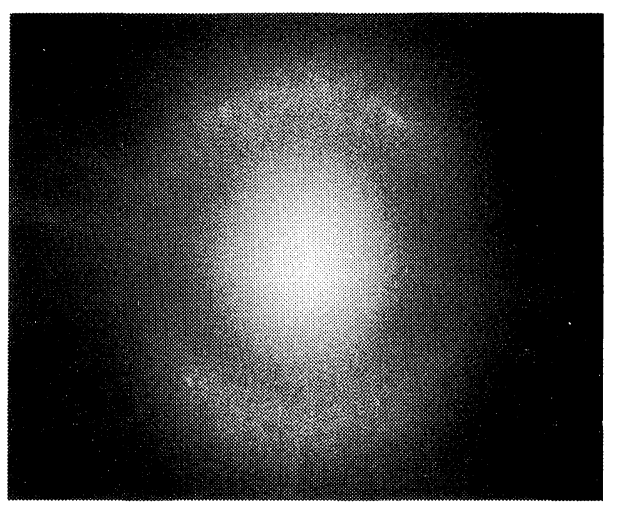

$H_{\mathrm{H}}$

Figure 3. $H_{\mathrm{V}}, V_{\mathrm{H}}, V_{\mathrm{V}}$, and $H_{\mathrm{H}}$ light scattering patterns from $16.9 \mathrm{wt} \%$ dioxane solution of $\mathrm{PBLG}$ at room temperature.

crystals. The concentration used here is in accord with the conventional definition, i.e., weight of polymer divided by that of solution. The dark and bright fringes in the micrograph reflect the twisting of $\alpha$-helical molecules in the cholesteric liquid crystals. ${ }^{10}$ The spacing $S$ between the successive fringes corresponds to half the cholesteric pitch $P$, i.e., the distance over which the optical axes make one complete turn.

Regular helicoidal twisting of the optical axes in the cholesteric texture gives rise to the optical diffraction shown in Figure 3, and the scattering intensity becomes maximum at the scattering angle $\theta_{\mathrm{m}}{ }^{\prime}$ satisfying the Bragg's eq 1 . Figure 3 shows typical "cholesteric scattering" with maximum in- tensity at $0 \simeq 7.5^{\circ}$ (the scattering angle in air) under $H_{\mathrm{V}}, V_{\mathrm{V}}, V_{\mathrm{H}}$, and $H_{\mathrm{H}}$ polarization conditions where the symbols $A$ and $B$ in $A_{\mathrm{B}}$ denote the polarization directions of the analyzer and the polarizer, respectively, and $H$ and $V$ denote the horizontal and vertical polarization, respectively. The depolarized components of the cholesteric scattering (i.e., the $H_{\mathrm{V}}$ and $V_{\mathrm{H}}$ scattering) are maximum at odd multiples of the azimuthal angle $\mu=45^{\circ}\left(\mu=0\right.$, and $90^{\circ}$ being taken along meridian and equator), since the cholesterics with the directors $\boldsymbol{n}$ (a unit vector along the molecular axis) oriented at $\pm 45^{\circ}$ directions with respect to the polarizer and analyzer axes have maximum induced dipole moments. On the other hand the polarized components of the cholesteric 


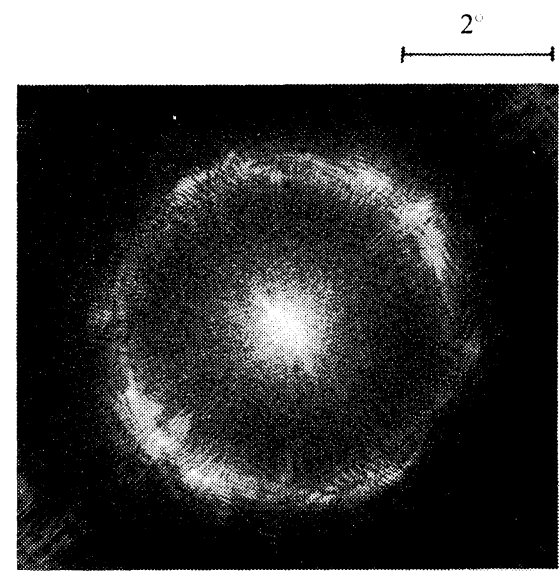

$V_{\mathbf{H}}$

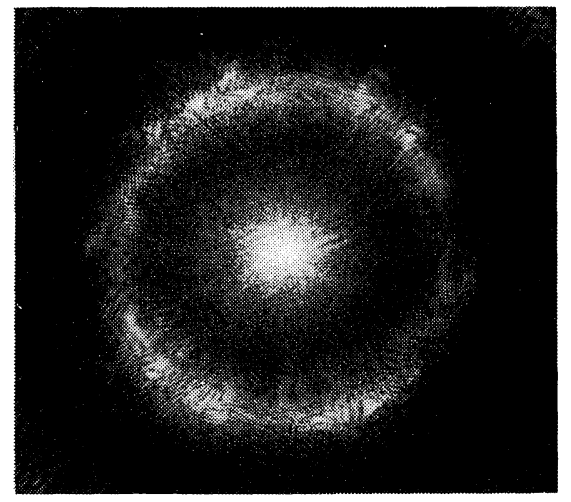

$H_{\mathrm{H}}$

Figure 4. $H_{\mathrm{V}}, V_{\mathrm{H}}, V_{\mathrm{V}}$, and $H_{\mathrm{H}}$ light scattering patterns from $17.5 \mathrm{wt} \% m$-cresol solution of PBLG at room temperature.

scattering have maximum intensities at $\mu=90$ and $270^{\circ}$, i.e., at equator, for $V_{\mathrm{v}}$ and at $\mu=0$ and $180^{\circ}$, i.e., at meridian, for $H_{\mathrm{H}}$, since the cholesterics with the directors $n$ oriented in vertical and horizontal directions have maximum induced dipole moments under $V_{\mathrm{v}}$ and $H_{\mathrm{H}}$ polarization conditions, respectively.

Close observation of the depolarized pattern shown in Figure 3, however, shows an anomaly in the $\mu$-dependence of the "cholesteric scattering"; the intensities at $\mu=45$ and $225^{\circ}$ are weaker than those at $\mu=135$ and $315^{\circ}$ under $H_{\mathrm{V}}$ polarization. This distortion of the $H_{\mathrm{V}}$ pattern is a mirror image of that of the $V_{\mathrm{H}}$ with respect to meridian; the $V_{\mathrm{H}}$ scattering intensities at $\mu=45$ and $225^{\circ}$ are stronger than those at $\mu=135$ and $315^{\circ}$. Thus we can write

$$
I_{H_{v}}(q, \mu)=I_{V_{h}}(q,-\mu)
$$

or

$$
I_{H_{v}}(q, \mu)=I_{V_{h}}\left(q, \mu+90^{\circ}\right)
$$

The distortion in the $H_{\mathrm{V}}$ and $V_{\mathrm{H}}$ patterns strongly suggests that it originates from an effect of the optical activity on the light scattering, as will be discussed, in detail, in THEORETICAL ANALYSIS section. ${ }^{18}$ Moreover from the discussions in THEORETICAL ANALYSIS section, one can see that the distortion of the "cholesteric pattern" is attributed to an extraordinary large "form-optical" rotatory power of the cholesteric liquid crystals ${ }^{7,11,12}$ and that the sense of the distortion of the depolarized light scattering patterns is 


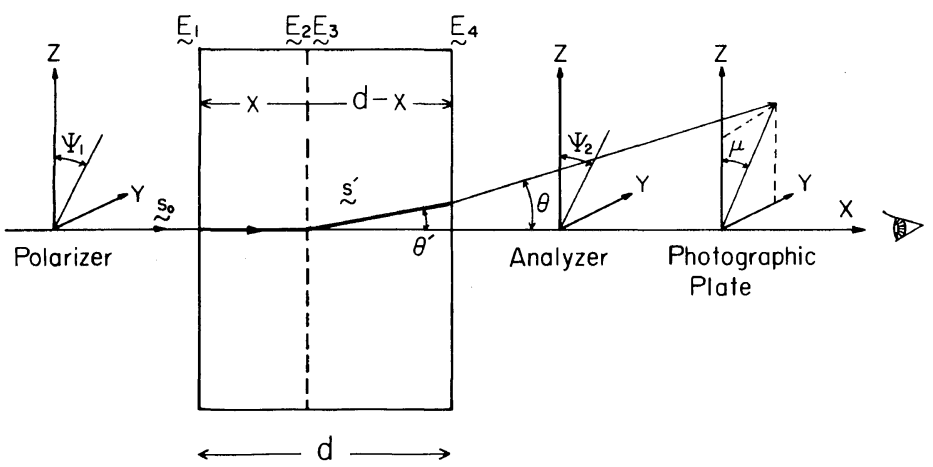

Figure 5. Optical set-up in the scattering experiment, and the definitions of the angles $\psi_{1}, \psi_{2}, \mu, \theta$, and $\theta$.

determined by the sign of the rotatory power, i.e., the sense of the cholesteric twisting. ${ }^{22}$ The manner of the distortion in the depolarized patterns of Figure 3 indicates that the twisting for the PBLG in $16.9 \mathrm{wt}^{\mathrm{o}} \%$ dioxane solution is rightrhanded, as will be shown later in THEORETICAL ANALYSIS section.

Figure 4 shows the depolarized and polarized components of scattering from a $17.5 \mathrm{wt} \%$ PBLG solution in $m$-cresol at room temperature. The $H_{\mathrm{V}}$ and $V_{\mathbf{H}}$ patterns are distorted in a manner similar to that in Figure 3, indicating right-handed cholesteric twisting. The $\mu$-dependences of $V_{\mathrm{V}}$ and $H_{\mathrm{H}}$ patterns are also similar to those in Figure 3, except for the fact that the angular dependencies are somewhat obscured due to "speckling" of the scattering pattern in comparison with those in Figure 3. This pronounced speckling effect may be attributed to intra- and inter-particle interferences of "cholesteric domain"13 (i.e., the region in which the helical axes of the cholesteric twisting have common orientation). This is obviously related to the size of the "cholesteric domain." The greater the size of the domain relative to the size of irradiated volume of sample, the more pronounced is the speckling effect. It may be worth noting that the cholesteric pitch $P$ in Figure 4 is much larger than that in Figure 3.

The $H_{\mathrm{V}}$ "cholesteric scattering" from the TCE solutions gave the same sense of distortion as that in Figures 3 or 4 ; these solutions thus have righthanded cholesteric twisting, while the twisting in the DCE solution had a sense of distortion opposite to those in Figures 3 and 4. The DCEsolution thus has negative rotatory power and left-handed cholesteric twisting. ${ }^{6}$ The sense of distortion in the depolarized "cholesteric scattering pattern" depends not only on the solvent but also on temperature as dem- onstrated in Figure 6 of ref 6 , and as shown in Figures 10 and 11 .

It also depends on concentration as shown in Figure 10. The reversal in the sense of cholesteric twisting with temperature, and its dependence on solvents and concentrations provide basic information for understanding physics of cholesteric twisting. The investigation of this, however, is beyond the scope of this paper.

\section{THEORETICAL ANALYSES}

In this section, we present a theoretical interpretation of the $\boldsymbol{q}$ dependence of "cholesteric scattering."

\section{Model}

Figure 5 shows an optical set-up for the scattering experiment. The incident beam is plane-polarized with its electric vector oriented at an angle $\psi_{1}$ from the vertical direction. The scattered beam is recorded through an analyzer with its electric vector oriented at an angle $\psi_{2}$ by a two-dimensional detector as a function of $\boldsymbol{q}=(q, \mu), \mu$ being the azimuthal angle. The angles $\psi_{1}, \psi_{2}$, and $\mu$ are taken to be positive when an observer, facing the incident beam, sees the clockwise rotation of the respective vectors, according to the conventional definition in optics. The sample with thickness $d$ is placed in between the polarizer and analyzer.

It is well known that cholesteric liquid crystals possess extraordinarily high optical rotatory power, called "form optical rotation" that arises from the twisted structure of the cholesteric liquid crystals $^{7,11,12}$ rather than from the intrinsic optical 
activity of $\alpha$-helical molecules. Owing to the effect of this form optical rotation, the electric vector $\boldsymbol{E}_{1}$ of a plane-polarized incident beam is rotated into $\boldsymbol{E}_{2}$ while it traverses over a distance $x$ before reaching the scattering element. The electric vector $\boldsymbol{E}_{3}$ of the scattered light is also subjected to a rotation into $\boldsymbol{E}_{4}$ before it leaves the sample. Under this condition where the effect of optical activity on the scattering is significant, the scattered intensity distributions cbserved under $V_{\mathrm{H}}$ and $H_{\mathrm{V}}$ polarizations should be affected by the polarized components of the scattered light, resulting in the distortion of the depolarized patterns.

The angle $\rho$ by which the electric vector of the incident beam is rotated, after travelling the distance $x$, is given by

$$
\rho=K x
$$

where $K$ is the parameter which defines the optical ratatory power of the cholesteric liquid crystal. According to the conventional optical definition, we define $K$ to be positive when an observer, facing the incident beam sees the clockwise rotation of the electric vector. $\boldsymbol{E}_{2}$ is thus given by,

$$
\boldsymbol{E}_{2}=E_{0}\left[\sin \left(\psi_{1}+\rho\right) \hat{\boldsymbol{y}}+\cos \left(\psi_{1}+\rho\right) \hat{z}\right]
$$

where $E_{0}$ is the field strength of the incident light, and $\hat{y}$ and $\hat{z}$ are unit vectors along $y$ and $z$ axes, respectively.

In the case in which cholesteric liquid crystal forms a homogeneous medium having constant optical properties (such as cholesteric pitch $P$ and the birefringence $\Delta n$ ) and a perfect orientation of the helical axis $\left(\hat{z}_{1}\right.$ in Figure 6$)$ of the cholesteric twisting in a direction parallel to the propagation direction of the incident beam, $K$ is simply given by, ${ }^{7.10}$

$$
K=-(\Delta n)^{2} P /\left(4 \lambda_{0}^{2}\right)
$$

if the cholesteric pitch $P$ is much larger than the wavelength of the incident light, as in our experiment. In eq $5, \lambda_{0}$ is the wavelength of light in vacuum. However in the analyses of THEORETICAL ANALYSIS sections 3, and $4, K$ is treated as an adjustable parameter which describes the "effective" optical rotatory power of the medium for a given cholesteric domain, and so eq 5 is not used. This is because in real systems, the orientation of the helical axes $\hat{z}_{1}$ are not perfect but more or less random. Orientational disorders

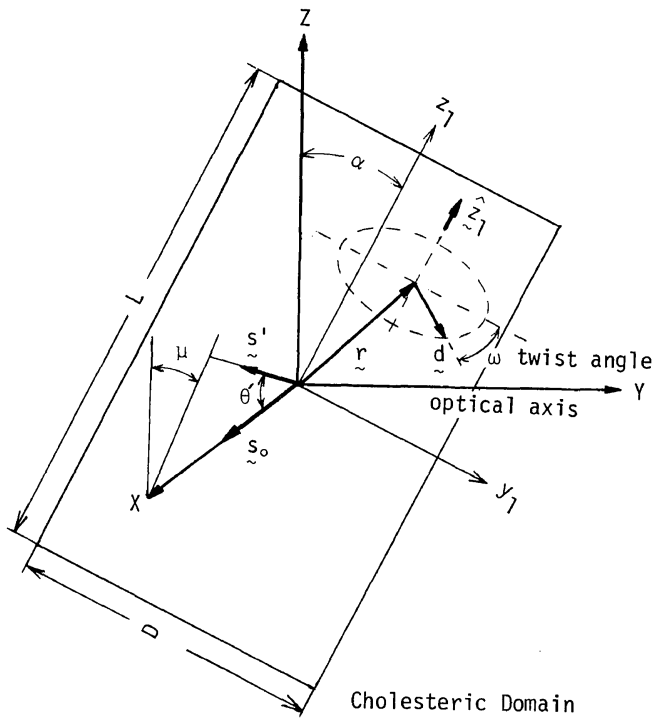

Figure 6. Scattering model of the domain of the cholesteric liquid crystal.

should result in an effective $K$ having a value much less than that predicted by eq. 5 .

It should be noted that the positive (right-handed or clockwise) optical rotatory power of cholesteric liquid crystals (i.e., $K>0$ ) indicates the right-handed twisting of the optical axes in the cholesteric liquid crystals. ${ }^{14}$

The "cholesteric domain" in which the helical axes of the cholesteric twisting (as designated by unit vector $\hat{z}_{1}$ in Figure 6) have a common orientation is assumed to have a rectangular parallelpiped shape of length $L$ along the twisting axis $\hat{z}_{1}$ and lateral width $D$ along the $y_{1}$ axis and $T$ along the $x_{1}$ axis, as shown in Figure 6. The optical axis as designated by unit vector $\boldsymbol{d}$ (identical to the director $\boldsymbol{n}$ in the literature of the liquid crystals ${ }^{15}$ ) is oriented normal to $\hat{z}_{1}$ and twisted helicoidally around the axis $\hat{z}_{1}$. The twist angle $\omega$ is a function of only $z_{1}$, giving rise to alternating bright and drak fringes perpendicular to $\hat{z}_{1}$ with the spacing $S=P / 2$, under the polarized light microscope.

In this paper we simplify the problems of calculating the "cholesteric scattering" by assuming $\hat{z}_{1}$ to be randomly oriented in a plane $Y O Z$, normal to the incident beam. This assumption hardly affects the generality of the conclusions drawn in this work. Moreover from the phase factor, $\exp [i(\boldsymbol{q} \cdot \boldsymbol{r})]$, in the scattering equation we can state that the orientation 
correlation in the plane $Y O Z$ normal to incident beam makes the greatest contribution to the angular dependence of the scattered light with respect to $q$, compared to the orientation correlation in any other planes (e.g., the orientation correlation in $X O Z$ plane makes negligible contribution to the angular dependence of the scattered light since $\boldsymbol{q} \cdot \boldsymbol{r} \simeq 0$ under this circumstance). The generalized treatment will be presented in a forthcoming paper and be based on the three dimensional random orientation of the cyclindrical "cholesteric domain." 13

\section{Elastic Scattering from the "Cholesteric} Domains",

In this section, we first calculate elastic scattering from a single cholesteric domain with a given orientation as specified by an angle $\alpha$ in Figure 6 embedded in an optically inactive, isotropic medium. ${ }^{23}$ In THEORETICAL ANALYSIS section 3 , we generalize the treatment so as to account for optical activity of the medium.

In the context of the Born approximation, the scattering amplitude $A$ in the Fraunhofer limit (i.e., far-field amplitude) is given by

$$
A=C \int \mathrm{d} \boldsymbol{r}(\boldsymbol{M} \cdot \boldsymbol{O}) \exp [i(\boldsymbol{q} \cdot \boldsymbol{r})]
$$

where $C$ is the constant related to absolute intensity, and

$$
\boldsymbol{q}=(2 \pi / \lambda)\left(s^{\prime}-s_{0}\right)
$$

$\boldsymbol{s}^{\prime}$ and $s_{0}$ are the unit vectors along the propagation directions of the scattered ray and ircident ray, respectively (see Figure 6).

$$
\begin{aligned}
\boldsymbol{s}_{0}=\hat{\boldsymbol{x}} & \\
\boldsymbol{s}^{\prime}=(\cos \theta) \hat{\boldsymbol{x}} & +(\sin \theta \sin \mu) \hat{\boldsymbol{y}} \\
& +(\sin \theta \cos \mu) \hat{\boldsymbol{z}}
\end{aligned}
$$

where $\hat{\boldsymbol{x}}, \hat{\boldsymbol{y}}$, and $\hat{\boldsymbol{z}}$ are unit vectors along $x, y$, and $z$ axes. $\boldsymbol{O}$ is a unit vector along the electric vector of the analyzer and is given by

$$
\begin{gathered}
\boldsymbol{O}_{\mathrm{V}}=\left(-\sin \rho_{1}\right) \hat{\boldsymbol{x}}+\left(\cos \rho_{1}\right) \hat{\boldsymbol{z}} \\
\text { for vertical polarization } \\
\boldsymbol{O}_{\mathrm{H}}=\left(-\sin \rho_{2}\right) \hat{\boldsymbol{x}}+\left(\cos \rho_{2}\right) \ddot{\boldsymbol{y}}
\end{gathered}
$$$$
\text { for horizontal polarization }
$$

where $^{16}$

$$
\begin{aligned}
& \cos \rho_{1}=\cos \theta^{\prime}\left[\cos ^{2} \theta^{\prime}+\sin ^{2} \theta^{\prime} \cos ^{2} \mu\right]^{-1 / 2} \\
& \cos \rho_{2}=\cos \theta^{\prime}\left[\cos ^{2} \theta^{\prime}+\sin ^{2} \theta^{\prime} \sin ^{2} \mu\right]^{-1 / 2}
\end{aligned}
$$

The induced dipole moment $\boldsymbol{M}$ depends on the orientation of the optical axis $\boldsymbol{d}$, orientation of the electric vector of the polarizer (whose unit vector is designated as $\boldsymbol{e}$ ) and optical indicatrices of the scattering element, (assumed to have uniaxial anisotropy with polarizabilities $\alpha_{1}$ and $\alpha_{2}$ parallel and perpendicular to the optical axes $d$, respectively),

$$
\boldsymbol{M}=E_{0}\left[\delta(\boldsymbol{e} \cdot \boldsymbol{d}) \boldsymbol{d}+b_{\mathrm{t}} \boldsymbol{e}\right]
$$

where $\delta=\left(\alpha_{1}-\alpha_{2}\right)$, optical anisotropy, and $b_{t}=$ $\alpha_{2}-\alpha_{\mathrm{s}}, \alpha_{\mathrm{s}}$ being the polarizability of the medium. $\boldsymbol{e}$ is equal to $\hat{z}$ for the vertically polarized incident beam and $\hat{\boldsymbol{y}}$ for horizontally polarized beam. $\boldsymbol{d}$ is given by,

$\boldsymbol{d}=(\sin \omega) \hat{\boldsymbol{x}}+(\cos \omega \cos \alpha) \hat{\boldsymbol{y}}-(\cos \omega \sin \alpha) \hat{\boldsymbol{z}}$

where $\omega$ is the twist angle, varying with $z_{1}$. For the homogeneous twisting of the optical axis,

$$
\omega=\omega\left(z_{1}\right)=(2 \pi / P) z_{1}
$$

In the forthcoming paper we shall consider the effect of inhomogeneous twisting, i.e., nonlinear variation of $\omega$ with $z_{1} \cdot{ }^{13}$ We shall treat $\delta$ and $b_{\mathrm{t}}$ as constants within the domain, i.e., the domain is homogeneous with $\delta$ and $b_{t}$.

From eq 6 to 16, the scattering amplitude for a given domain oriented at an angle $\alpha, A(\alpha)$, is given by

$$
\begin{aligned}
A(\alpha)= & C D T j_{0}\left(\frac{\pi T}{\lambda}\left(1-\cos \theta^{\prime}\right)\right) \\
& \times j_{0}\left(\frac{\pi D}{\lambda} \sin \theta^{\prime} \sin (\alpha-\mu)\right) \\
& \times \int_{-L / 2}^{L / 2} \mathrm{~d} z_{1}(\boldsymbol{M} \cdot \boldsymbol{O}) \\
& \times \exp \left[-\frac{2 \pi}{\lambda} z_{1} \sin \theta^{\prime} \cos (\alpha-\mu)\right] \\
\simeq & C D T j_{0}\left(\frac{1}{2} q D \sin (\alpha-\mu)\right) \\
& \times \int_{-L / 2}^{L / 2} \mathrm{~d} z_{1}(\boldsymbol{M} \cdot \boldsymbol{O}) \exp \left[-q z_{1} \cos (\alpha-\mu)\right]
\end{aligned}
$$


where $j_{0}(x)$ is the zeroth-order spherical Bessel function of the first kind. The $H_{\mathrm{V}}$ scattering at relatively small scattering angles, $A_{H_{v}}(\alpha)$, is given by, ${ }^{24}$

$$
\begin{gathered}
A_{H_{v}}(\alpha)=-C_{1} B(\alpha, \mu) F(\alpha, \mu) \sin 2 \alpha \\
C_{1}=(1 / 8) L E_{0} C D \delta \\
B(\alpha, \mu)=j_{0}\left(\frac{1}{2} q D \sin \theta^{\prime} \sin (\alpha-\mu)\right)
\end{gathered}
$$

$$
\begin{aligned}
F(\alpha, \mu)= & \left\{2 j_{0}(g W)+j_{0}((g+m) W)+j_{0}((g-m) W)\right. \\
& -\frac{1}{2} i\left[2 g W j_{0}{ }^{2}\left(\frac{1}{2} g W\right)\right. \\
& +(g+m) W j_{0}{ }^{2}\left(\frac{1}{2}(g+m) W\right) \\
& \left.\left.+(g-m) W j_{0}{ }^{2}\left(\frac{1}{2}(g-m) W\right)\right]\right\}
\end{aligned}
$$

by noticing that

$$
\begin{aligned}
& \cos \rho_{1}=\cos \rho_{2} \cong 1, \sin \rho_{1}=\sin \rho_{2} \simeq 0 \\
& (\boldsymbol{M} \cdot \boldsymbol{O})_{H_{\mathrm{v}}}=-\frac{1}{2} E_{0} \delta \sin 2 \alpha \cos ^{2} \omega\left(z_{1}\right)
\end{aligned}
$$

at small $q^{\prime}$ s. $q, W$, and $m$ in eq 21 are defined as

$$
\begin{aligned}
& W=q L \\
& m=\lambda /\left(2 S \sin \left(\theta^{\prime} / 2\right)\right) \\
& g=\cos (\alpha-\mu)
\end{aligned}
$$

Thus the scattered intensity $\left(\left|A_{H_{v}}(\alpha)\right|^{2}\right)$ becomes maximum at $g= \pm m$, i.e.,

$$
2 S \cos (\alpha-\mu) \sin \left(\theta^{\prime} / 2\right)=\lambda
$$

where $S \cos (\alpha-\mu)$ is the "effective identity period" of the cholesteric twisting for a given $q$. The scattered intensity at $\mu=\alpha$ becomes maximum at the scattering angle $\theta_{\mathrm{m}}$ ' satisfying eq 1 . It is very important to note that homogeneous twisting gives rise only to the first-order scattering maximum, but to no higher order maxima.

Similarly, the scattering amplitudes under other polarization conditions can be calculated,

$$
\begin{gathered}
A_{V_{h}}(\alpha)=A_{H_{v}}(\alpha) \\
A_{H_{h}}(\alpha)=2 K B(\alpha, \mu)\left[F(\alpha, \mu) \cos ^{2} \alpha+p G(\alpha, \mu)\right] \\
A_{V_{v}}(\alpha)=2 K B(\alpha, \mu)\left[F(\alpha, \mu) \sin ^{2} \alpha+p G(\alpha, \mu)\right]
\end{gathered}
$$

$$
G(\alpha, \mu)=4 j_{0}(g W)-2 i g W j_{0}^{2}\left(\frac{1}{2} g W\right)
$$

and,

$$
p=b_{\mathrm{t}} / \delta=\left(\alpha_{2}-\alpha_{\mathrm{s}}\right) /\left(\alpha_{1}-\alpha_{2}\right)
$$

\section{Effect of Form Optical Rotation}

Now we generalize the problem in THEORET-

ICAL ANALYSES section 2 for a case in which a single cholesteric domain is embedded in a homogeneous medium having the effective optical rotatory power $K$ as defined by eq 3 .

The effect of the optical rotation on the elastic scattering was first explored by Stein and $\operatorname{Picot}^{9}$ on spherulitic scattering. We can adopt the same principle as that established by Stein and Picot, remarking that their amplitudes $A_{V_{v}}, A_{H_{h}}, A_{H_{v}}$, and $A_{V_{h}}$ are constant, indepent of $\alpha$, but that our amplitudes are dependent on $\alpha$, thus giving rise to an important difference in the calculation technique of these two cases.

The horizontal and vertical component of the scattered field $\left(E_{3}\right)_{H}$ and $\left(E_{4}\right)_{H}$ are given, in terms of the scattered amplitudes $A_{H_{h}}, A_{H_{v}}, A_{V_{v}}$, and $A_{V_{h}}$, by

$$
\begin{aligned}
& \left(E_{3}\right)_{H}=A_{H_{h}}(\alpha) \sin \left(\psi_{1}+\rho\right)+A_{H_{v}}(\alpha) \cos \left(\psi_{1}+\rho\right) \\
& \left(E_{3}\right)_{V}=A_{V_{v}}(\alpha) \cos \left(\psi_{1}+\rho\right)+A_{V_{h}}(\alpha) \sin \left(\psi_{1}+\rho\right)
\end{aligned}
$$

The plane of polarization of the scattered field $\boldsymbol{E}_{3}$ is again rotated when it traverses the medium to result in $\boldsymbol{E}_{4}$, the horizontal and vertical components being given by

$$
\begin{aligned}
& \left(E_{4}\right)_{H}=\left(E_{3}\right)_{V} \sin \rho^{\prime}+\left(E_{3}\right)_{H} \cos \rho^{\prime} \\
& \left(E_{4}\right)_{V}=\left(E_{3}\right)_{V} \cos \rho^{\prime}-\left(E_{3}\right)_{H} \sin \rho^{\prime}
\end{aligned}
$$

where

$$
\rho^{\prime}=K(d-x) / \cos \theta^{\prime}
$$

$K$ is the effective optical rotatory power of the cholesterics as defined by eq 3 . Thus the scattering amplitude transmitted by an analyzer oriented at an angle $\psi_{2}$ as in Figure 5, $E_{\Psi_{1}, \Psi_{2}}$, is given by,

$$
\begin{gathered}
E_{\Psi_{1}, \Psi_{2}}=\boldsymbol{E}_{4} \cdot \boldsymbol{O}^{\prime} \\
\boldsymbol{O}^{\prime}=\sin \psi_{2} \hat{\boldsymbol{y}}+\cos \psi_{2} \hat{z}
\end{gathered}
$$

From eq 31 to 37 , if follows that

$$
\begin{aligned}
E_{H_{v}}(\alpha, x) & =E_{\Psi_{1}=0, \Psi_{2}=90} \\
& =\left[A_{V_{v}}(\alpha) \cos \rho+A_{V_{h}}(\alpha) \sin \rho\right] \sin \rho^{\prime}
\end{aligned}
$$


Supramolecular Structure of Polypeptides. II.

$$
+\left[A_{H_{h}}(\alpha) \sin \rho+A_{H_{v}}(\alpha) \cos \rho\right] \cos \rho^{\prime}
$$

The average scattered intensity $I_{H_{v}}$ is given by,

$$
\begin{aligned}
I_{H_{v}}= & \frac{1}{2 \pi d} \int_{0}^{d} \mathrm{~d} x \int_{0}^{2 \pi} \mathrm{d} \alpha E_{H_{v}}(\alpha, x) E_{H_{v}}^{*}(\alpha, x) \\
= & \left|A_{H_{v}}\right|^{2} \Omega_{1}+\left|A_{V_{h}}\right|^{2} \Omega_{2}+\left|A_{H_{h}}\right|^{2} \Omega_{3}+\left|A_{V_{v}}\right|^{2} \Omega_{4} \\
& +2 \operatorname{Re}\left(A_{V_{v}} A_{H_{h}}^{*}+A_{H_{v}} A_{V_{h}}^{*}\right) \Omega_{5} \\
& +2 \operatorname{Re}\left(A_{H_{v}} A_{H_{h}}^{*}\right) \Omega_{6}+2 \operatorname{Re}\left(A_{H_{v}} A_{V_{v}}^{*}\right) \Omega_{7} \\
& +2 \operatorname{Re}\left(A_{V_{h}} A_{H_{h}}^{*}\right) \Omega_{8}+2 \operatorname{Re}\left(A_{V_{h}} A_{V_{v}}^{*}\right) \Omega_{9}
\end{aligned}
$$

where the terms $\left|A_{H_{v}}\right|^{2}, \operatorname{Re}\left(A_{V_{v}} A_{H_{h}}^{*}\right)$ and etc. are obtained by numerical integration of the respective quantities with $\alpha, e . g$.,

$$
\left|A_{H_{v}}\right|^{2}=\frac{1}{2 \pi} \int_{0}^{2 \pi} \mathrm{d} \alpha A_{H_{v}}(\alpha) A_{H_{v}}^{*}(\alpha)
$$

while the terms like $\Omega_{1}$ are obtained following integration of the corresponding terms with $x, e . g$.,

$$
\Omega_{1}=\frac{1}{d} \int_{0}^{d} \mathrm{~d} x \cos ^{2} \rho \cos ^{2} \rho^{\prime}
$$

The terms $\Omega_{i}$ have already been calculated by Stein and Picot and are equal to $\Omega_{i}{ }^{\prime}$ in eq 24 of reference 9. Needless to say, eq 39 is based on the assumption of independent scattering from the cholesteric domains. Similary, $V_{\mathrm{H}}$ scattering intensity is given by,

$$
\begin{aligned}
I_{V_{h}}= & \left|A_{V_{h}}\right|^{2} \Omega_{1}+\left|A_{H_{v}}\right|^{2} \Omega_{2}+\left|A_{V_{v}}\right|^{2} \Omega_{3}+\left|A_{H_{h}}\right|^{2} \Omega_{4} \\
& +2 \operatorname{Re}\left(A_{H_{v}} A_{V_{h}}^{*}+A_{H_{h}} A_{V_{v}}^{*}\right) \Omega_{5} \\
& -2 \operatorname{Re}\left(A_{V_{v}} A_{V_{h}}^{*}\right) \Omega_{6}-2 \operatorname{Re}\left(A_{V_{h}} A_{H_{h}}^{*}\right) \Omega_{7} \\
& -2 \operatorname{Re}\left(A_{V_{v}} A_{\mathrm{H}_{v}}^{*}\right) \Omega_{8}-2 \operatorname{Re}\left(A_{H_{h}} A_{H_{v}}^{*}\right) \Omega_{9}
\end{aligned}
$$

Noticing that at small $\theta, \Omega_{3} \simeq \Omega_{4}, \Omega_{6} \simeq \Omega_{7}$, and $\Omega_{8} \simeq \Omega_{9}$, it follows that,

$$
\begin{aligned}
I_{H_{v}}(q, \mu ;-K) & =I_{H_{v}}(q, \mu+90 ; K) \\
& =I_{V_{h}}(q, \mu ; K)
\end{aligned}
$$

Thus, the distortion of the $H_{\mathrm{V}}$ or $V_{\mathrm{H}}$ pattern depends on the sign of $K$, i.e., the sense of cholesteric twisting. The $V_{\mathrm{H}}$ pattern is obtained by a $90^{\prime \prime}$ rotation of the $H_{\mathrm{v}}$ pattern around the incident beam axis, as found in the experimental patterns shown in Figures 3 and 4.

\section{Results}

Numerical calculations of the elastic scattering from the cholesteric domains are performed for a given set of the parameters; $L / S=16$ and $D / L=2$ as a function of $K d$ and $p=\left(\alpha_{2}-\alpha_{\mathrm{s}}\right) /\left(\alpha_{1}-\alpha_{2}\right)$. The value of $D / L=2$ is arbitrarily chosen, but the conclusions drawn hereafter are completely independent of this choice.

Figure 7 shows the calculated $H_{\mathrm{v}}$ scattering patterns from the cholesteric twisted structure for $p=-1 / 3$. When $K d=0^{\circ}$, the medium is optically inactive, and the form optical rotation has no effect on the scattering pattern, so that the $H_{\mathrm{v}}$ pattern has a fourfold symmetry in terms of the $\mu$-dependence, with maximum intensity at odd multiples of $\mu=45^{\circ}$. The contour patterns are plotted as a function of the reduced scattering vector $W$ (eq 22) and azimuthal angle $\mu$. The "cholesteric scattering" has a maximum intensity at $\theta_{\mathrm{m}}$ ' satisfying the Bragg equation 1 and consequently at $W_{\mathrm{m}}$ satisfying

$$
W_{\mathrm{m}}=q_{\mathrm{m}} L=2 \pi(L / S)=32 \pi \simeq 100
$$

as seen in Figure 7.

The $X$-type small angle scattering pattern at $W \ll 80$ reflects the scattering from the cholesteric domain as a whole, its angular dependencies with $\mu$ and $W$ being a function of $D$ and $L$, the dimensions of the domain. The greater the shape anisotropy of the domain, the larger the $\mu$-dependence of the scattering. The larger the dimensions, the more the scattering appears at small $q$. The small-angle scattering, designated as "rod-like scattering," is throughly discussed in our previous paper. ${ }^{8}$

The greater the value of $K d$, i.e., the greater the effect of the form optical rotation, the more distorted is the $\mu$-dependence of the pattern. When the medium has a positive optical rotatory power (i.e., $K>0$ ), the intensity at $\mu=45$ and $225^{\circ}$ is weaker than that at $\mu=135$ and $315^{\circ}$. The small-angle rodlike scattering patterns are also distorted but their distortions are opposite to those for the "cholesteric scattering" patterns. ${ }^{17}$

Figure 8 shows the effect of changing the sign of $K$ on the $H_{\mathrm{v}}$ scattering pattern for the same set of the parameters as those in Figure 7. It is shown that the negative $K$, i.e., the negative optical rotatory power of the medium, gives rise to the pattern distorted in direction opposite to the pattern for the 


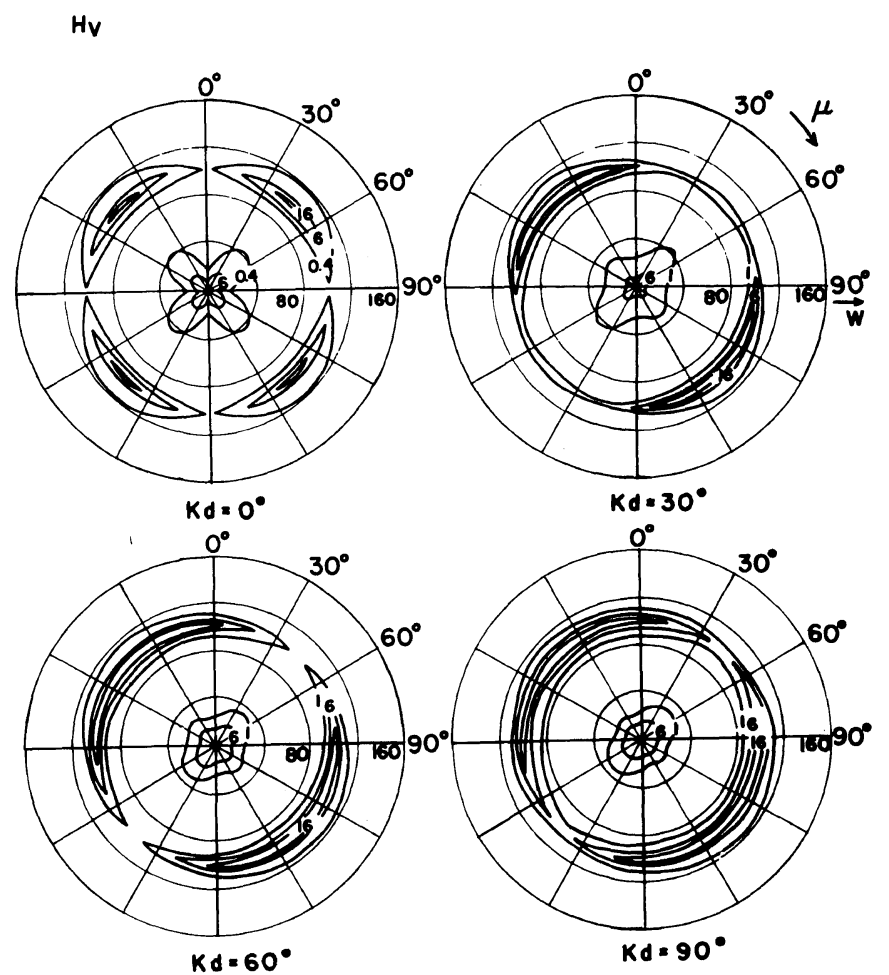

Figure 7. Calculated $H_{\mathrm{v}}$ scattering pattterns from the cholesteric twisted structure as a function of the optical activity $K d ; L / S=16, D / L=2$, and $p=-1 / 3$.
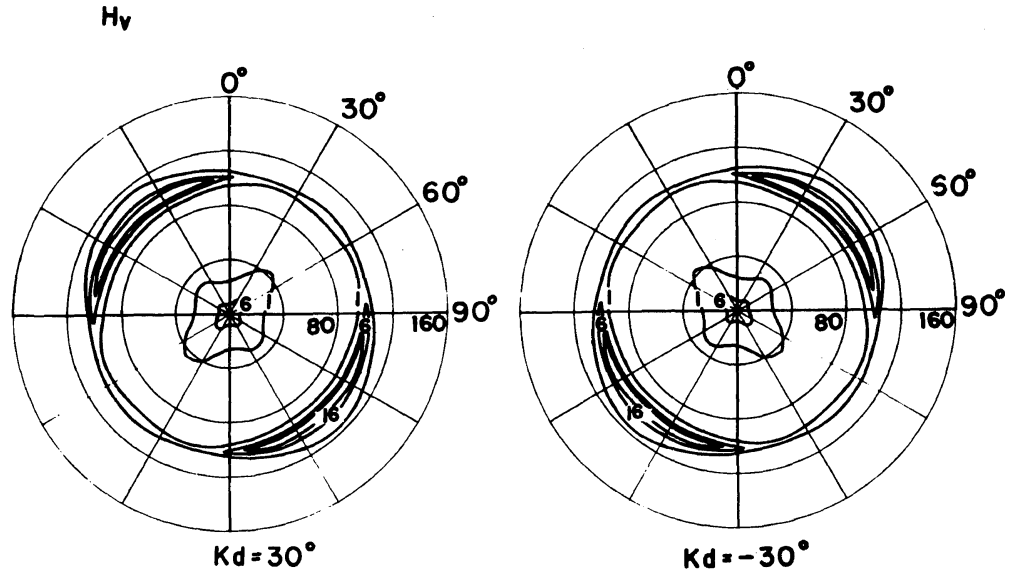

Figure 8. Effect of sign $K$ (optical rotatory power) on $H_{\mathrm{v}}$ scattering pattern; $L / S=16, D / L=2$, and $p=-1 / 3$.

positive $K$. This result confirms eq 43 and provides a method for determining the sense of the optical rotatory power and therefore the sense of the cholesteric twisting.
Figure 9 shows the effect of changing the parameter $p=\left(\alpha_{2}-\alpha_{\mathrm{s}}\right) /\left(\alpha_{1}-\alpha_{2}\right)$ on the $H_{\mathrm{V}}$ scattering pattern for the same set of parameter as in Figures 7 and 8, i.e., $L / S=16, D / L=2$, and $K d=60^{\circ}$. 

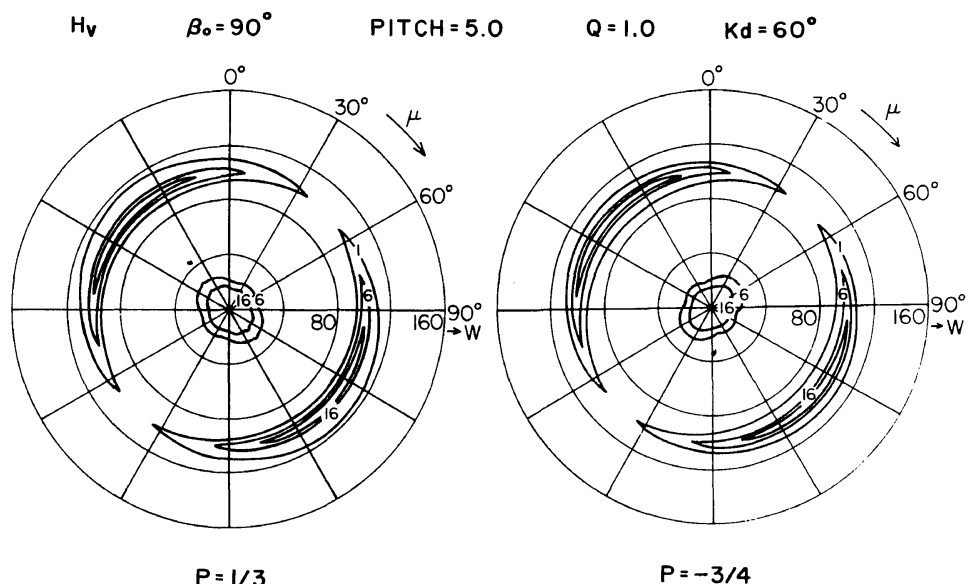

Figure 9. Effect of the parameter $p=\left(\alpha_{2}-\alpha_{\mathrm{s}}\right) /\left(\alpha_{1}-\alpha_{2}\right)$ on $H_{\mathrm{v}}$ scattering pattern; $L / S=16, D / L=2$, and $K d=60$.

Although the parameter $p$ affects the "rod-like scattering" appearing at small $q$, the "cholesteric scattering at large $q$ is hardly affected by the parameter $p$. This is quite reasonable in view of the fact that the "rod-like scattering" depends not only on the spatial orientation correlation but also on the density correlation, and is consequently dependent of the parameter $p$. However the "cholesteric scattering" depends only on the orientation correlation, and is consequently independent of the parameter $p$. Thus the sign of the rotatory power can be unambiguously determined from the distortion of the depolarized "cholesteric scattering" pattern. However the value $p$ is prerequisite for estimating the sign from the depolarized "rod-like scattering."

\section{DISCUSSION}

According to the principles established in EXPERIMENTAL section and THEORETICAL ANALYSIS section, we estimated the spacing $S$ (from eq 1) and the sense of cholesteric twisting. We take $S$ to be positive when $K$ is positive, i.e., the cholesteric system has positive rotatory power and right-handed twisting according to Uematsu et al. ${ }^{14}$

Figures 10 and 11 show plots of reciprocal $S$ (associated with the helical twisting power of the cholesterics) against temperature for the PBLG-TCE system and PBLG- $m$-cresol system, respectively. The scattering maximum of the depolarized "cholesteric pattern" was observed to shift toward smaller angles with increasing tempera-

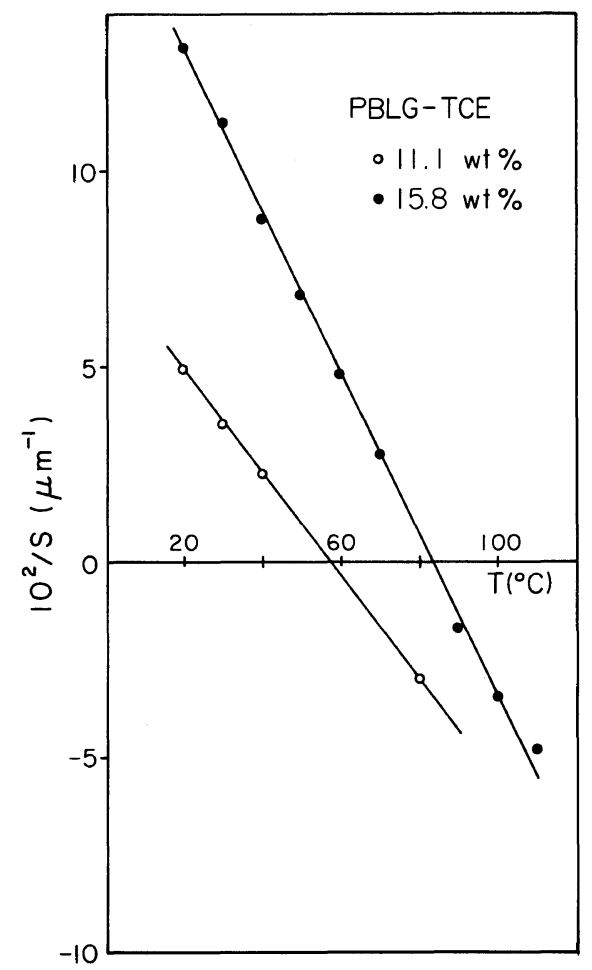

Figure 10. Plot of $1 / S$ (helical twisting power of the cholesterics) against temperature for 11.1 and $15.8 \mathrm{wt} \%$ TCE solutions of PBLG.

ture up to $T_{N}$ at which the maximum disappears. With a further increase in temperature the scattering maximum was observed to appear again and to shift toward larger scattering angles. Thus, the 


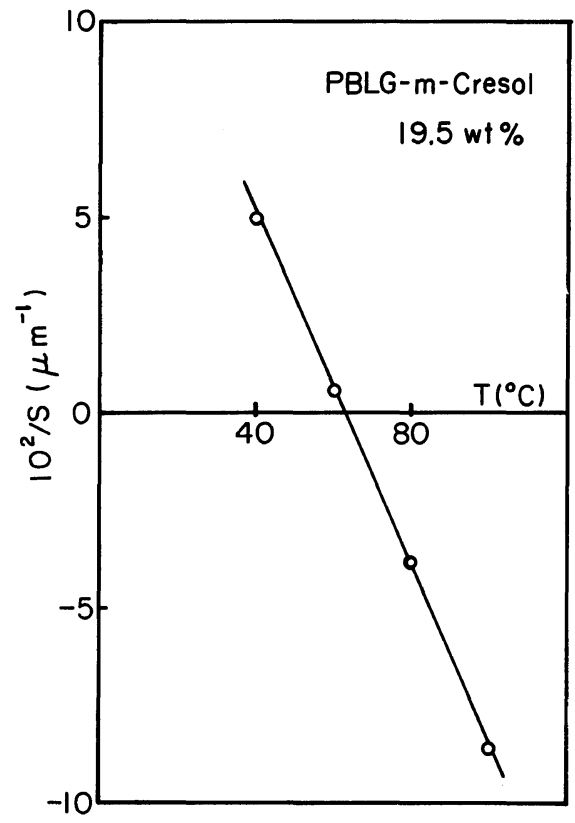

Figure 11. Plot of $1 / S$ against temperature for $19.5 \mathrm{wt} \% m$-cresol solution of PBLG.

cholesteric pitch increases and approaches infinity at $T_{N}$ with increasing temperature. The pitch decreases with a further increase in temperature. The patterns are distorted in opposite directions below and above $T_{N}$, reflecting the change in sign $K$ from positive to negative.

These results indicate that the temperature $T_{N}$ is a function of polymer concentration (Figure 10 for PBLG-TCE system) and solvent (polymer-solvent interaction, cf. Figures 10 and 11). The reversal of the sign demonstates that there are two counterbalancing forces causing the twisting. The investigations of this, however, is beyond the scope of this work. The negative sign of $S$ for the PBLG-DCE system at room temperature ${ }^{6}$ may result from the fact that $T_{N}$ for this system is lower than room temperature. The simultaneous estimations of the sense and the pitch of the cholesteric twisting thus help to provide a systematic understanding of the physics underlying in this twisted system.

\section{REFERENCES AND NOTES}

1. C. Robinson, Trans. Faraday Soc., 52, 571 (1955).

2. I. Uematsu, Kobunshi, 27, 754 (1978).

3. K. Ito, T. Kajiyama, and M. Takayanagi, Polym. J.,
12, 305 (1980).

4. J. Watanabe, K. Imai, and I. Uematsu, Polym. Bull., 1, 67 (1978).

5. E. Iizuka, Polym. J., 4, 401 (1973).

6. T. Hashimoto, S. Ebisu, and H. Kawai, J. Polym. Sci., Polym. Lett. Ed., 18, 569 (1980).

7. H. L. de Vries, Acta Cryst., 4, 219 (1951).

8. T. Hashimoto, T. Ijitsu, K. Yamaguchi, and H. Kawai, Polym. J., 12, 745 (1980).

9. C. Picot and R. S. Stein, J. Polym. Sci., A-2, 8, 1491 (1970).

10. C. Robinson, Molecular Cryst., 1, 467 (1966).

11. G. Friedel, Ann. Phys. (Paris), 18(9), 273 (1922).

12. J. Mathieu, J. Bull. Soc. Trans. Minerol., 34, 13 (1911).

13. T. Hashimoto, N. Inaba, S. Ebisu, and H. Kawai, Polym. J., 13 (1981) in press.

14. H. Toriumi, Y. Kusumi, Y. Uematsu, and I. Uematsu, Rep. Prog. Polym. Phys. Jpn., 21, 569 (1978), Polym. J., 11, 863 (1979).

15. see for example, P. G. deGennes, "The Physics of Liquid Crystals," Clarendon, Oxford, 1974.

16. S. Clough, J. J. van Aartsen, and R. S. Stein, J. Appl. Phys., 36, 3072 (1965).

17. This is possible since the "cholesteric scattering" depends only on the spatial orientation correlation of the optical axes, while the "rodlike scattering" depends as well on the density correlation owing to the effect of optical activity of the medium, (thus the sense of distortion of the "rodlike scattering pattern" should also depend on $p$ ).

18. The distortion of the patterns also arises from a macroscopic orientation of the cholesteric domains as pointed out in ref 6 . One can distinguish two kinds of distortions, at least, in principle. If the distortion purely arises from the effect of form optical rotation, the pattern under given polarization condition is unchanged with the rotation of the sample around the incident beam axis, while if the distortion arises from the macroscopic orientation, the pattern changes with the rotation due to the effect of birefringence on the scattering distribution. ${ }^{19-21}$ Moreover, if the distortion dominantly arises from the macroscopic orientation, the $H_{\mathrm{v}}$ pattern should be identical to the $V_{\mathrm{H}}$ pattern, while if the distortion arises from the optical activity, the $H_{\mathrm{V}}$ pattern is not identical to, but related to the $V_{\mathrm{H}}$ pattern by eq 2 .

19. W. Chu and R. S. Stein, J. Polym. Sci., A-2, 8, 489 (1970).

20. M. Motegi, M. Moritani, and H. Kawai, J. Polym. Sci., A-2, 8, 499 (1970).

21. N. Hayashi, Y. Murakami, M. Moritani, T. Hashimoto, and H. Kawai, Polym. J., 4, 560 (1973).

22. If the orientation of the cholesteric domains significantly contributes to the distortion of the pattern, the determination of the sense of the cholesteric twisting is somewhat obscured. 
Supramolecular Structure of Polypeptides. II.

23. In a real system, a given cholesteric domain is surrounded by other cholesteric domains, so that there may be no isotropic phase as assumed in our model. Thus, the surrounding medium is generally anisotropic, having polarizabilities $\alpha_{\mathrm{s} 1}$ and $\alpha_{\mathrm{s} 2}$ parallel and perpendicular to their optical axes. In this treatment we assume the medium has an average polarizability $\alpha_{s}=\left(\alpha_{s 1}+\alpha_{s 2}\right) / 2$. This assumption is good for our systems in which scattering dominantly arises from anisotropy fluctuations (or orientation fluctuations) rather than from density fluctuations caused by difference in the average polarizabilities between the cholesteric domain and the surrounding medium.

24. It should be noted that at small scattering angles, $j_{0}\left(\pi T\left(1-\cos \theta^{\prime}\right) / \lambda\right) \rightarrow 1$ unless $T$ is extraordinarily large compared with $L$ and $D$. 